$\phi\left\{f_{1}\left(t_{1}, t_{2}, \cdots, t_{n}, x_{1}\right), f_{2}\left(t_{1}, t_{2}, \cdots, t_{n}, x_{2}\right), \cdots\right.$,

$$
\left.f_{n+1}\left(t_{1}, t_{2}, \cdots, t_{n}, x_{n+1}\right)\right\}=\psi\left(x_{1}, x_{2}, \cdots, x_{n+1}\right) \text {, }
$$

where $n=1,2,3$, etc. From this class of equations other classes are deduced, first, by the homologous transposition of the $x$ 's in the left hand member and, second, by substituting $x$ 's for some or all of the $t$ 's in the set of equations thus obtained (including the original class). For example, one obtains by homologous transposition of the $x$ 's when $n=2$

$$
\begin{aligned}
& \phi\left\{f_{1}\left(x_{1}, t_{1}, t_{2}\right), f_{2}\left(x_{2}, t_{1}, t_{2}\right), f_{3}\left(x_{3}, t_{1}, t_{2}\right)\right\}=\psi\left(x_{1}, x_{2}, x_{3}\right) \\
& \phi\left\{f_{1}\left(t_{1}, x_{1}, t_{2}\right), f_{2}\left(t_{1}, x_{2}, t_{2}\right), f_{3}\left(t_{1}, x_{3}, t_{2}\right)\right\}=\psi\left(x_{1}, x_{2}, x_{3}\right) .
\end{aligned}
$$

Interesting problems are presented by systems of equations belonging to the same or different classes.

22. The proposition that the accelerations of different bodies under the action of equal forces are inversely proportional to their masses is often asserted to be merely a definition of mass. The object of the paper by Professor Hoskins is to show that, in applying this proposition, our interpretation of it involves the notion of mass as a quantitative measure of the matter of which the bodies are composed.

23. Dr. Bennett considers several topics connected with the iteration of functions of one variable. Matrices with an infinite number of elements are used to obtain a classification of the types of series which are to be distinguished with respect to the nature of their iteration. The different types are considered, with particular reference to questions of convergence. The iteration of functions of a real variable is also considered. The paper will appear in the Annals of Mathematics.

\title{
GROUPLESS TRIAD SYSTEMS ON FIFTEEN ELEMENTS.
}

BY DR. LOUISE D. CUMMINGS AND PROFESSOR H. S. WHITE.

(Read before the American Mathematical Society April 24, 1915.)

From previous publications and a paper presented to this Society in October, 1914, 44 different triad systems on 15 elements $\left(\Delta_{15}\right)$ are known. These 44 systems separate into two types 23: of the systems each contain one or more systems 
on 7 elements and have been designated as headed systems, the remaining 21 contain no $\Delta_{7}$ and have been designated as headless systems. The groups for the 44 systems have been obtained and range in order from $8 ! / 2$ down to 2 . Hence for each of these systems there exists at least one substitution different from identity which transforms the system into itself.

Systems whose group is identity or groupless systems on 15 elements have not been known hitherto. No groupless systems exist for 7, 9, or 13 elements. In a paper published in the Transactions, January, 1915, one of us has proved the existence of millions of triad systems on 31 elements with the group identity. This raised the question concerning the existence of the groupless systems for 15 elements. About six months ago we began a rigorous search for this type of $\Delta_{15}$, and, while the investigation is at present far from completion, we have had the good fortune to discover systems of this new species.

In the Transactions for January, 1913, Professor Cole discussed triad systems on 13 elements, and pointed out the fact that two elements in a system may be connected by what he has termed an "interlacing"; for example the four triads $a 12$, $a 34, b 13, b 24$ occurring in a system form an interlacing of the elements $a$ and $b$. Interlacing and some extensions of this idea have proved to be of fundamental importance in the formation of the groupless systems.

All interlacings and the more extended forms of interconnection of the elements, which we make use of, may be obtained by applying to the system under consideration what may be called a contracted form of sequences and indices. The $\Delta_{15}$ is written in a 15 by 7 array. Each element heads one column; below it are placed the 7 pairs of elements which with the element at the head occur in the triads of the system. Heretofore, sequences and indices have been formed from the 3 columns of a triad in any $\Delta_{15}$. The same process is now applied to every pair of columns of a $\Delta_{15}$. Since the number of combinations of 15 columns, two at a time, is 105 , 105 pairs of columns must be examined, unless the group for the system is already known and is different from identity. If the group contains an operator of order $k$, then $k$ pairs of columns are examined simultaneously. The process may be illustrated in its application to a system $J$, with a group of order 4 generated by $s=(a)(b c)(d 8 e 7)(f 3 g 4)(1526)$. Pairs of 
columns selected from the following table show every type of contracted index that occurs in any system.

\begin{tabular}{|c|c|c|c|}
\hline$a$ & $b$ & $d$ & 1 \\
\hline$d e$ & $d f$ & $a e$ & $a 2$ \\
$f g$ & $e g$ & $b f$ & $b 3$ \\
12 & $a c$ & $c 2$ & $c e$ \\
34 & 13 & $g 8$ & $d 5$ \\
56 & 24 & 15 & $f 8$ \\
78 & 57 & 37 & $g 4$ \\
$b c$ & 68 & 46 & 67 \\
\hline
\end{tabular}

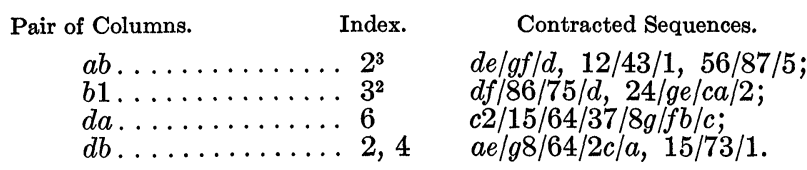

The substitution $s$ applied to the pair of columns $a b$ gives the pair $a c$ with the same index and similar sequences. If $s$ is applied to the pair of columns $b 1$, the three pairs $c 5, b 2, c 6$ are obtained with the index $3^{2}$. The analysis of the 105 pairs of columns shows that the contracted indices $2^{3} ; 2,4 ; 3^{2} ; 6$ belong, respectively, to $2,24,4$, and 75 pairs of the columns.

The new groupless systems are formed by interchanging duads of one column with those of another column. For example, in the pair of columns $a b$, the duads $d e, f g$ of column $a$ may be interchanged with $d f$, eg of column $b$; such an interchange involving four elements, contained only in two pairs in each column, shall be designated as a quadrangular transformation. The columns $d, e, f, g$ must now be re-written, in agreement with the new triads introduced into the system, and the undisturbed 9 columns of $J$ with the re-constructed 6 columns form a new system $J Q$. The four duads 12, 34, 56,78 of column $a$ might be interchanged with the four duads on the same elements in column $b$, forming an octagonal transformation, but this is equivalent to the above quadrangular transformation followed by the interchange of the elements $a$ and $b$.

In the pair of columns $b 1$ the duads $d f, 68,57$ of column $b$ may be interchanged with the duads $f 8,67, d 5$ of column 1 ; such an interchange, involving six elements appearing exclusively in 3 pairs in each of the two columns, shall be designated as a hexagonal transformation. New columns $d, f, 6,8,5,7$ 
must next be constructed; these eight reconstructed columns, with the seven undisturbed columns of $J$, form a system $J H$. The application of the second hexagonal transformation in $b 1$ is equivalent to an application of the first hexagonal transformation followed by the interchange of the elements $b$ and 1 .

A transformation on 12 elements simply interchanges the two elements which head the columns.

Therefore only the quadrangular and the hexagonal transformations which exist in a system require consideration.

By means of the operators of the group of the system, the minimum number of non-congruent transformations of each of the above types is determined-for example, in $J$, the 8 hexagonal transformations reduce to 1 , and the 30 quadrangular transformations to 4 non-congruent transformations.

Each of the non-congruent transformations is now applied to the system $J$, and the sequences and indices are determined for the five transformed systems.

The 35 triads of the system $J H$ arranged in classes according to their indices are shown in the following table.

\begin{tabular}{|c|c|c|c|c|c|c|c|c|}
\hline $1^{4} 26$ & $1^{4} 35$ & $1^{3} 2^{2} 5$ & $1^{3} 9$ & $1^{2} 2^{3} 4$ & $1^{228}$ & $1^{2} 37$ & $1^{2} 46$ & $1^{2} 5^{2}$ \\
\hline 157 & $\begin{array}{l}a 12 \\
258\end{array}$ & $\begin{array}{l}a 78 \\
a 56 \\
c g 5\end{array}$ & 168 & $a 34$ & $\begin{array}{l}g 14 \\
c 47 \\
d g 8\end{array}$ & $c d 2$ & $b 24$ & $b 13$ \\
\hline $12^{27}$ & 1236 & 1245 & 111 & $2^{2} 4^{2}$ & $23^{24}$ & 5,7 & $6^{2}$ & \\
\hline $\begin{array}{l}\text { ade } \\
d 46\end{array}$ & $\begin{array}{l}a f g \\
g 27\end{array}$ & $\begin{array}{l}e 26 \\
f 45 \\
c f 6 \\
c 38 \\
d 37\end{array}$ & $\begin{array}{l}b d 5 \\
b f 8 \\
b 67 \\
b e g \\
c e 1\end{array}$ & $\begin{array}{l}e 48 \\
d f 1 \\
g 36\end{array}$ & ef7 & $\begin{array}{l}e 35 \\
f 23\end{array}$ & $a b c$ & \\
\hline
\end{tabular}

The enumeration of the entrances of the elements in the 17 classes shows that the transitive sets of elements are $a$; $b$; $c ; d ; e ; f ; g ; 1 ; 2 ; 3 ; 4 ; 5 ; 6 ; 7 ; 8$; hence the group for the system is identity. Therefore under the hexagonal transformation the system $J$, with a group of order 4 , is changed into a system $J H$ with the group identity. The four quadrangular transformations applied to $J$ yield four non-congruent systems. One of these is a new groupless system $J Q$; the remaining three are known headless systems with groups of orders 2,2 , and 6 respectively. 
The work of transforming the 44 known systems has been partially performed and we have discovered about 20 systems of the new groupless type. The headed type of $\Delta_{15}$ is exhausted in 23 systems; the non-headed with a group, in 21 . The numerous repetitions now appearing in the work lead us to believe that the groupless type is not much more numerous than the other two types. The purpose of the present investigation is to obtain, if possible, a complete set of groupless systems, and then to show the connecting links between all the systems of the three types.

The system $J H$ with no head and no group, which has been derived by a hexagonal transformation from a system $J$, with no head, but with a group of order 4, may also be derived from another system $I C$ with a head and a group of order 3 . Hexagonal transformations leave unchanged the number of $\Delta_{7}$ 's in a $\Delta_{15}$, and, therefore, always transform systems with or without heads into systems with or without heads respectively. A quadrangular transformation may change the number of $\Delta_{7}$ 's in the $\Delta_{15}$. The indices of the new groupless systems show a rather marked difference from the indices of the previously known systems. It has been found convenient to list the indices of systems, so that those indices which contain the greatest number of sequences of period 1 appear at the lefthand side of the index table; that is, the indices are written in descending order of sequences of period 1. Systems with a group contain indices of the types $1^{12}, 1^{8} 2^{2}$,- but no groupless system has yet been found with an index containing more than six sequences of period 1 -for example, $1^{6} 3^{2}$ contains the maximum number of sequences of period 1 found among the indices of the groupless systems. Hence the indices of the groupless type show a distinct shifting towards the right-hand side of the index table. This shift was to be expected since the new systems are headless and, therefore, the indices necessarily involve fewer sequences of period 1 .

Vassar College. 